\title{
A critical evaluation of drug delivery from ligand modified nanoparticles: confounding small molecule distribution and efficacy in the central nervous system
}

Rebecca L. Cook ${ }^{\mathrm{a}, \mathrm{b}}$, Kyle T. Householder ${ }^{\mathrm{a}, \mathrm{b}}$, Eugene P. Chung ${ }^{\mathrm{a}, \mathrm{c}}$, Alesia V. Prakapenka, ${ }^{\mathrm{a}, \mathrm{d}}$, Danielle M. DiPerna $^{a}$, Rachael W. Siriannia ${ }^{a, b, d^{*}}$

\author{
AFFILIATIONS \\ ${ }^{a}$ Barrow Brain Tumor Research Center, Barrow Neurological Institute, 350 West Thomas Road, Phoenix \\ AZ 85013. \\ ${ }^{\mathrm{b}}$ School of Biological and Health Systems Engineering, Ira A. Fulton Schools of Engineering, Arizona \\ State University, P.O Box 879709, Tempe, AZ, 85287, USA \\ ${ }^{\mathrm{c}}$ School of Life Sciences, Arizona State University, P.O Box 874701, Tempe, AZ, 85287, USA \\ ${ }^{\mathrm{d}}$ Interdisciplinary Graduate Program in Neuroscience, School of Life Sciences, Arizona State University, \\ P.O Box 874701, Tempe AZ 85287
}

EMAIL ADDRESSES

RLC: rebeccalmccall@gmail.com

KTH: kth19@ case.edu

EPC: epchung@asu.edu

AVP: aprakapenka@gmail.com

DMD: dannidiperna@gmail.com

RWS: rachael.sirianni@ dignityhealth.org

CORRESPONDING AUTHOR

*Rachael W. Sirianni

Neuroscience Research Center, room 436

St. Joseph's Hospital and Medical Center

350 W Thomas Road

Phoenix, AZ 85013

Rachael.sirianni@dignityhealth.org

KEYWORDS

Brain; Chemotherapy; Drug Delivery; Nanoparticle; Peptide; Spinal Cord

WORD COUNT

6,384 


\section{ABSTRACT}

In this work, we sought to test how surface modification of poly(lactic-co-glycolic acid) (PLGA) nanoparticles with peptide ligand alters the brain specific delivery of encapsulated molecules. For biodistribution studies, nanoparticles modified with rabies virus glycoprotein (RVG29) were loaded with small molecule drug surrogates and administered to healthy mice by lateral tail vein injection. Mice were perfused two hours after injection and major anatomical regions of the CNS were dissected (striatum, midbrain, cerebellum, hippocampus, cortex, olfactory bulb, brainstem, and cervical, thoracic, lumbar and sacral spinal cord). For functional studies, surface modified nanoparticles were loaded with the chemotherapeutic camptothecin (CPT) and administered to mice bearing intracranial GL261-Luc2 gliomas. Outcome measures included tumor growth, as measured by bioluminescent imaging, and median survival time. We observed that small molecule delivery from PLGA nanoparticles varied by as much as $150 \%$ for different tissue regions within the CNS. These differences were directly correlated to regional differences in cerebral blood volume. Although the presence of RVG29 enhanced apparent brain delivery for multiple small molecule payloads, we observed minimal evidence for targeting to muscle or spinal cord, which are the known sites for rabies virus entry into the CNS, and enhancements in brain delivery were not prolonged due to an apparent aqueous instability of the RVG29 ligand. Furthermore, we have identified concerning differences in apparent delivery kinetics as measured by different payloads: nanoparticle encapsulated DiR was observed to accumulate in the brain, whereas encapsulated Nile red was rapidly cleared. Although systemically administered CPT loaded nanoparticles slowed the growth of orthotopic brain tumors to prolong survival, the presence of RVG29 did not enhance therapeutic efficacy compared to control nanoparticles. These data are consistent with a model of delivery of hydrophobic small molecules to the brain that does not rely on internalization of polymer nanoparticles in target tissue. We discuss an important risk for discordance between biodistribution, as typically measured by drug surrogate, and therapeutic outcome, as determined by clinically relevant measurement of drug function in a disease model. These results pose critical considerations for the methods used to design and evaluate targeted drug delivery systems in vivo.

\section{INTRODUCTION}

Drug delivery remains a critical barrier to the treatment of central nervous system (CNS) tumors such as glioblastoma (GBM). The access of systemically administered drugs to cells residing beyond the primary tumor mass remains poor, and peripheral toxicity often limits total dose. A multitude of reports suggest that encapsulation of small molecules in nanoparticles can improve their delivery or function in the CNS [1-6], however, whether these improvements are due to actual movement of the nanoparticle across an intact blood-brain barrier (BBB) into the parenchyma remains unclear.

In the context of cancer, non-specific tumor targeting can sometimes be achieved by the enhanced permeability and retention (EPR) effect, whereby nanoparticles circulating in peripheral vasculature are believed to exit leaky, angiogenic tumor vasculature to selectively accumulate in tumor core [7-9]. Surface modification of nanoparticles with targeting ligands has been proposed as a method for enhancing delivery of nanoparticle encapsulated payloads to specific tissue sites, including tumor or brain. Recently, a peptide derived from rabies virus glycoprotein emerged as a promising candidate for brain-targeted drug delivery. RVG29 is the 29 amino acid fragment of the rabies virus coat protein whose presence is both necessary and sufficient for the interaction of virus with cell surface receptors to promote retrograde transport of virus along motor neurons into the brain. RVG29 has been reported to govern the interaction of viral particles with at least three known receptors, including neural cell adhesion molecule (NCAM), the p75 neurotrophin receptor (P75NTR), and nicotinic acetylcholine receptors (NAChRs) $[10,11]$. NAChRs were suggested to be responsible for the transvascular delivery 
of nucleic acids tethered to an RVG29-9R conjugate[12], although a recent report studying the uptake of RVG29-tethered dendrimers provided evidence for the involvement of gamma-aminobutyric acid B receptors $\left(\mathrm{GABA}_{\mathrm{B}} \mathrm{Rs}\right)$ instead [13]. The RVG29 peptide has been demonstrated to enhance the brainspecific function of a range of systemically delivered agents, particularly nucleic acids [12, 14-17] and has also been used to facilitate the CNS efficacy of nanoscale drug and gene carriers, including proteins and biologically derived or synthetic nanoparticles [18-25]. Notably, RVG29-mediated delivery of small molecule therapeutics remains relatively unexplored [24].

In prior work, we demonstrated that systemically administered nanoparticles composed of PLGA and loaded with the chemotherapeutic camptothecin (CPT) slowed the growth of orthotopic, murine GBM to prolong survival [8]. Improved therapy of nanoparticle encapsulated CPT was primarily mediated by EPR and increased dose due to enhanced tolerability of encapsulated drug. Here, we were interested to test how modification of PLGA nanoparticles with RVG29 would alter the delivery and efficacy of small molecule payloads in the brain.

\section{METHODS}

\section{Materials}

RVG29-biotin peptide (sequence: $\mathrm{N}$ term-YTIWMPENPRPGTPCDIFTNSRGKRASNG-C2-Biotin) was synthesized by American Peptide Company (Sunnyvale, CA, USA). 50:50 Poly(DL-lactide-coglycolide) (PLGA, ester terminated, 0.55 - 0.75 IV) was obtained from Durect Corporation (Cupertino, CA, USA). Reagents for nanoparticle preparation were purchased from Sigma-Aldrich (St. Louis, MO, USA). Cell culture reagents (DMEM, FBS, Geneticin) and DiR were purchased from Life Technologies (Carlsbad, CA, USA).

\section{Nanoparticle Preparation}

PLGA nanoparticles were prepared by the single emulsion method as described previously [8, 26]. Avidin-palmitate conjugation was performed as described elsewhere [27]. Briefly, 25mg of avidin was reacted with $1 \mathrm{mg}$ palmitic acid-NHS overnight while stirring at $37^{\circ} \mathrm{C}$ in $2 \% \mathrm{w} / \mathrm{v}$ sodium deoxycholate in PBS. The conjugate was purified by 48 hours of dialysis against $0.15 \% \mathrm{w} / \mathrm{v}$ sodium deoxycholate in PBS (MW cutoff of 14kDa). For dye loaded nanoparticles (biodistribution studies), 200mg of PLGA was dissolved in $2 \mathrm{~mL}$ dichloromethane $(\mathrm{DCM})$ with either $50 \mu \mathrm{L}$ of DiR in ethanol $(25 \mathrm{mg} / \mathrm{mL})$ or $100 \mu \mathrm{L}$ Nile red (NR) in DCM $(25 \mathrm{mg} / \mathrm{mL})$. This solution was added drop-wise to a vortexing aqueous phase containing $1 \mathrm{~mL} 5 \mathrm{mg} / \mathrm{mL}$ avidin-palmitate, $1 \mathrm{~mL} \mathrm{H}_{2} \mathrm{O}$, and $2 \mathrm{~mL} 5 \% \mathrm{w} / \mathrm{v}$ polyvinyl alcohol (PVA) to form an emulsion. The emulsion was sonicated in three, ten-second bursts (Fisher Scientific Model 705 Sonic Dismembrator, $40 \%$ amplitude) and hardened for three hours by stirring in $84 \mathrm{~mL} 0.3 \%$ PVA. Particles were washed twice in $\mathrm{diH}_{2} \mathrm{O}$ by centrifuging for 15 minutes at 17,400xg (Beckman L8-80M Ultracentrifuge, 50.2TI rotor). CPT nanoparticles were prepared using the same technique with the following parameters: $12 \mathrm{mg}$ of CPT was added to 200mg of PLGA in 2mL DCM and emulsified with an aqueous phase containing $1 \mathrm{~mL} 5 \mathrm{mg} / \mathrm{mL}$ avidin-palmitate, $3 \mathrm{~mL} \mathrm{H}_{2} \mathrm{O}$, and $4 \mathrm{~mL} 5 \%$ PVA.

\section{Nanoparticle Peptide Conjugation}

For surface modification, nanoparticles were incubated with a 10x molar excess of RVG29-biotin (RVG-PLGA) or biotin (Biotin-PLGA) for one hour. Nanoparticles were washed by centrifugation to remove excess ligand. A small sample was removed for scanning electron microscopy (SEM) characterization and trehalose (25mg per 100mg PLGA) was added to the remaining nanoparticle solution as a cryoprotectant. The nanoparticle solution was either aliquotted for immediate use for in vivo studies or frozen, lyophilized, and stored at $-80^{\circ} \mathrm{C}$. 


\section{Nanoparticle Characterization}

Nanoparticles were characterized for size and morphology with scanning electron microscopy (SEM, FEI XL30) and for hydrodynamic diameter with dynamic light scattering (DLS, NanoBrook 90Plus Zeta particle analyzer, Brookhaven Instruments, Hotsville, NY). Samples were prepared for SEM by sputter coating for 2 minutes with gold-palladium and imaged at a working distance of 5-15mm, 2-10kV, spot size of 1-5, and 10,000-40,000X magnification. Average nanoparticle size for each batch was calculated from SEM images using the measure function in ImageJ (National Institutes of Health) with a minimum of 150 measurements from two or more images. Polydispersity (PD) was calculated by taking the standard deviation of these measurements, or the mean standard deviation if more than one batch was prepared. For DLS measurements, average and PD were calculated by Particle Solutions Software (Brookhaven Instruments, Hotsville, NY). Loading of DiR, NR or CPT was determined by comparing nanoparticle samples dissolved in DMSO at a concentration of $5 \mathrm{mg} / \mathrm{ml}$ to a control curve prepared from blank nanoparticles spiked with known concentrations of dye or drug in DMSO. Loading was calculated by determining the weight of encapsulated molecule per nanoparticle batch, and dividing this value by the weight of PLGA (non-encapsulated molecule, non-trehalose weight) per batch.

To obtain a profile of CPT release from nanoparticles, RVG-PLGA-CPT nanoparticles with an equivalent mass of $6.5 \mu \mathrm{g}$ CPT were added to a total volume of $2 \mathrm{~mL}$ release medium (PBS $+2 \%$ Tween80 ) for each time point to be measured. This concentration assured sink conditions (reported solubility of CPT in PBS $+2 \%$ Tween- 80 is $79 \mu \mathrm{g} / \mathrm{mL}$ ). $[28,29]$ Samples were incubated while rotating at $37^{\circ} \mathrm{C}$. At each time point, corresponding samples were removed and centrifuged for ten minutes at 20,000xg. $30 \mu \mathrm{L}$ of supernatant was removed and mixed with $970 \mu \mathrm{L}$ release medium. CPT fluorescence was measured at an excitation/emission of $370 \mathrm{~nm} / 428 \mathrm{~nm}$ and compared to a standard of free CPT in release medium to determine concentration.

\section{Cell Culture}

GL261-Luc2 cells were maintained in DMEM supplemented with 10\% FBS and 100 $\mu \mathrm{g} / \mathrm{mL}$ Geneticin in a humidified chamber at $37^{\circ} \mathrm{C}$ with $5 \% \mathrm{CO}_{2}$. For tumor inductions, cells were washed, detached with trypsin, and centrifuged to obtain a pellet. Cells were resuspended in plain DMEM at a concentration of $3.75 \mathrm{e} 7$ cells $/ \mathrm{mL}$ for injection.

\section{Animal Studies}

All mice were housed in a 12:12 light dark cycle with food and water provided ad libitum. Nanoparticles were administered via injection into the lateral tail vein either as freshly prepared (treatment studies) or immediately following resuspension via sonication in sterile saline (biodistribution studies). All experimental procedures were performed in compliance with Barrow Neurological Institute's Institutional Animal Care and Use Committee (IACUC) regulations.

\section{Biodistribution}

Nanoparticles were administered at a dose of $200-250 \mathrm{mg} / \mathrm{kg}$ to $6-8$ week old female BalbC mice (Charles River Laboratories, USA). Subjects were sacrificed 2 hours post injection ( $\mathrm{n}=6$ per group), and a blood sample was obtained by cardiac puncture. Mice were perfused with heparinized saline $(10 \mathrm{U} / \mathrm{mL})$ until the livers cleared. Peripheral organs were removed, as well as whole brain, which was immediately dissected into major anatomical regions (olfactory bulb, cortex, striatum, midbrain, hippocampus, cerebellum, and brain stem) with fine pointed forceps under a dissecting microscope. Surgical scissors were also used to make a cut at the lower lumbar portion of the spine and a $1 \mathrm{~mL}$ syringe filled with saline attached to a $22 \mathrm{G}$ needle was inserted into the spinal canal opening. Spinal cords were rapidly ejected following applied pressure to the syringe plunger [30]. Spinal cords were dissected into its major anatomical parts (cervical, thoracic, lumbar, and sacral) using the spinal cord 
intumescences as guides. All samples were frozen at $-80^{\circ} \mathrm{C}$ until further processing. Values for cerebral blood volume in the mouse brain were obtained from published data for the mouse [31]. The cerebral blood volume value for brainstem was estimated by averaging the pons and medulla.

Peripheral organ tissue was thawed on ice and finely minced into a pulp. $10 \% \mathrm{w} / \mathrm{v} \mathrm{diH}_{2} \mathrm{O}$ was added to each sample. Tissue was physically disrupted in a bead homogenizer for 10 minutes at the highest speed and lysed by probe sonication (40\% amplitude for 10 seconds, $2 \mathrm{x}$ ) on ice. CNS tissue was processed by the same method, except samples were not bead homogenized. CNS homogenates $(40 \mu \mathrm{L})$ and peripheral organ homogenates $(50 \mu \mathrm{L})$ were added to a $96-$ well plate with $10 \mu \mathrm{L}$ of DMSO. Fluorescence intensity was read on a plate spectrophotometer (Tecan Infinite 2000, 750/780nm or 552/636nm excitation/emission for DiR or NR, respectively). To convert AU values to concentration, control curves were constructed for each organ by spiking control tissue homogenates with known quantities of DiRloaded nanoparticles. All samples were read in triplicate. In multiple experiments, we confirmed that there were no differences in control curve fluorescence for different CNS regions for tissue spiked either with free or nanoparticle encapsulated DiR (data not shown).

\section{Treatment Study}

The syngeneic GL261-Luc2 tumor model used in this study is an infiltrative model of GBM [32] that has been stably transfected with luciferase to enable noninvasive monitoring of tumor growth by bioluminescent imaging. Tumors were induced in the dorsal striatum of C57B1/6 albino mice as previously described [8]. Briefly, mice were anesthetized using a cocktail of ketamine (100 mg/kg) and xylazine $(10 \mathrm{mg} / \mathrm{kg})$ provided by intraperitoneal (IP) injection. An incision was made down the midline of the scalp to expose the skull and a hole was drilled $2 \mathrm{~mm}$ lateral, $0.1 \mathrm{~mm}$ posterior from bregma using a Dremel (Mount Prospect, IL). A Hamilton syringe filled with $2 \mu \mathrm{L}$ of the cell suspension (75,000 cells) was lowered to a depth of $3 \mathrm{~mm}$ and the tissue allowed to equilibrate for 1 minute. The syringe was then withdrawn to a depth of $2.6 \mathrm{~mm}$ and the cells injected over 2 minutes. After the injection, the syringe was left for 1 minute before it was removed to reduce back flow. The incision was closed using staples and triple antibiotic ointment was applied to the scalp before placing the animal in a clean cage over a heating pad to recover. All animals received a subcutaneous injection of buprenorphine SR (Reckitt Benckiser, Hull, England) $(0.2 \mathrm{mg} / \mathrm{kg})$ after surgery to control pain.

Weekly treatments were administered by lateral tail vein injection beginning on day 8 after tumor induction. Animals were randomized to four treatment groups: saline $(n=6)$, RVG-PLGA (n=5), BiotinPLGA-CPT $(\mathrm{n}=6)$, or RVG-PLGA-CPT $(\mathrm{n}=6)$. Drug treated mice received an average of $12 \mathrm{mg} / \mathrm{kg}$ CPT (RVG-PLGA-CPT or Biotin-PLGA-CPT) per injection. The RVG-PLGA group was dosed based on the average equivalent PLGA dose administered to Biotin-PLGA-CPT and RVG-PLGA-CPT groups. Mice were observed daily and euthanized following greater than $20 \%$ weight loss or development of neurological symptoms.

Tumor growth was monitored throughout the study using an In Vivo Imaging System (IVIS) bioluminescent imager (Perkin Elmer, Waltham, MA), a method which has been well-validated for the GL261-Luc2 model and allows for tumor detection by day four following tumor induction.[33] Mice were anesthetized in an induction chamber under $2 \%$ isoflurane in oxygen. Once anesthetized, mice were injected subcutaneously with $150 \mathrm{mg}$ luciferin/kg body weight and imaged 25 minutes later. Tumor size was measured by total flux (photons/second) as determined by the Living Image software for each region of interest (ROI) drawn around the tumor signal.

\section{Statistics}


Statistical analyses were conducted in GraphPad Prism 5.0 (GraphPad Software, Inc., La Jolla CA). Analysis of targeting to whole organs was conducted using a one-tailed Student's t-test. Regional analysis of biodistribution was conducted with a two-way, repeated measures (mixed model) ANOVA followed by a Student's t-test with a Bonferroni posthoc correction for multiple comparisons. Tumor growth profiles were fit by an exponential growth equation with ordinary least squares to estimate doubling time. Kaplan-Meier curves were constructed for treatment studies, using the Mantel-Cox test to probe for differences in survival. Significance is reported for an alpha level of 0.05 .

\section{RESULTS}

\section{Nanoparticle characterization and biodistribution}

SEM imaging confirmed that PLGA nanoparticles were spherical with smooth surface morphology (Figure 1). DiR, NR and CPT loaded nanoparticles were characterized by a similar average diameter (129-141nm, 111-132nm, and 134-142nm $\pm 10 \mathrm{~nm}$, respectively, Table 1). Loading, calculated as the average weight percent of encapsulated molecule in the final yield of PLGA, was $0.26 \%$ for DiR, $0.87 \%$ for NR, and $6.87 \%$ for CPT, with encapsulation efficiencies of $38.1 \%, 54.6 \%$, and $80.3 \%$, respectively. Surface charge was close to neutral. The rate of CPT release was determined by incubating RVGPLGA-CPT nanoparticles in PBS and centrifuging at regular time intervals to sample supernatant. A burst release was observed at early time points, with almost all remaining camptothecin released by 3 hours (Figure 2), whereas less than 5\% of NR or DiR was released in 24 hours (data not shown).
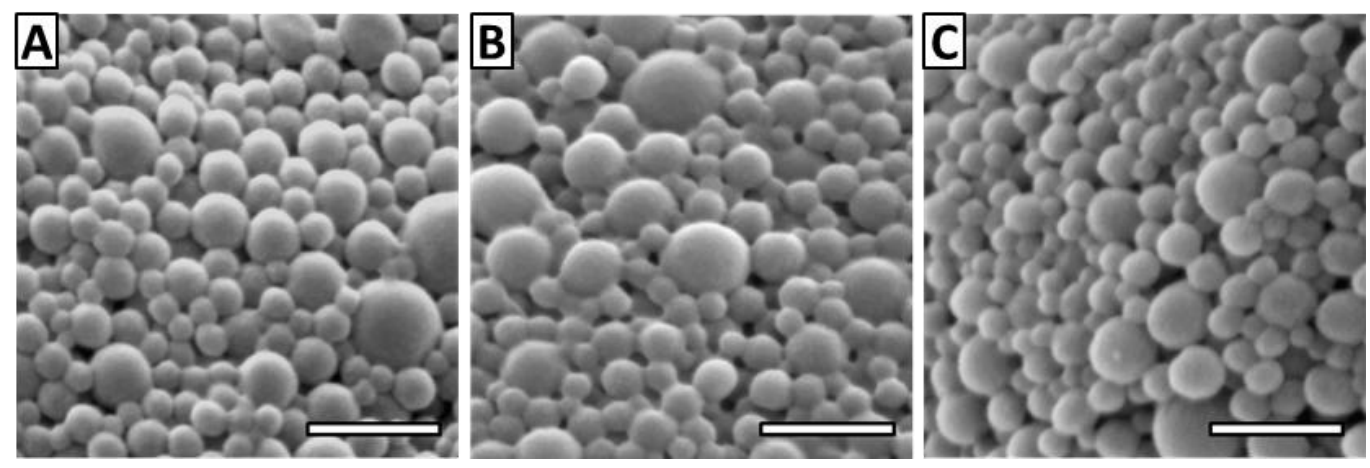

Figure 1: Nanoparticles were spherical and exhibited a smooth morphology. (A) RVG-PLGA-CPT and (B) Biotin-PLGA-CPT had an average diameter of 138 $\pm 10 \mathrm{~nm}$ ( $n=3$ batches). (C) RVG-PLGA nanoparticles had an average diameter of $156 \pm 36 \mathrm{~nm}$ ( $n=3$ batches). Standard deviation represents variation between batches. Scale bar $=500 \mathrm{~nm}$ 


\begin{tabular}{|c|c|c|c|c|c|c|c|c|}
\hline \multirow{4}{*}{$\begin{array}{l}\text { Name } \\
\text { RVG-PLGA-DiR } \\
\text { Biotin-PLGA-DiR }\end{array}$} & \multirow{4}{*}{$\begin{array}{c}\text { Payload } \\
\text { DiR }\end{array}$} & \multirow{4}{*}{$\begin{array}{c}\text { Loading } \\
0.26 \%\end{array}$} & \multirow{4}{*}{$\begin{array}{c}\mathbf{E E}^{\mathbf{l}} \\
38.1 \%\end{array}$} & \multicolumn{2}{|c|}{ SEM } & \multicolumn{2}{|c|}{ DLS } & \multirow{4}{*}{$\begin{array}{c}\text { Zeta Potential } \\
\text { (mV) } \\
0.36 \pm 1.76 \\
1.69 \pm 0.95\end{array}$} \\
\hline & & & & (nm) & $\mathbf{P D}^{3}$ & (nm) & $\begin{array}{l}\mathbf{P D}^{3} \\
\text { 1) }\end{array}$ & \\
\hline & & & & 129 & 36 & 188 & 44 & \\
\hline & & & & 141 & 31 & 238 & 56 & \\
\hline \multirow{2}{*}{$\begin{array}{l}\text { RVG-PLGA-NR } \\
\text { Biotin-PLGA-NR }\end{array}$} & \multirow{2}{*}{ NR } & \multirow{2}{*}{$0.87 \%$} & \multirow{2}{*}{$54.6 \%$} & 132 & 33 & 162 & 64 & $-0.88 \pm 2.98$ \\
\hline & & & & 111 & 37 & 182 & 40 & $-3.35 \pm 1.89$ \\
\hline RVG-PLGA-CPT & \multirow{2}{*}{$\mathrm{CPT}$} & \multirow{2}{*}{$6.87 \pm 0.9 \%$} & \multirow{2}{*}{$80.3 \pm 7 \%$} & $142 \pm 10$ & 47 & 204 & 45 & $-2.50 \pm 1.19$ \\
\hline Biotin-PLGA-CPT & & & & $134 \pm 10$ & 43 & 252 & 31 & $-2.18 \pm 1.19$ \\
\hline RVG-PLGA & None & N/A & N/A & $156 \pm 36$ & 64 & 253 & 69 & $-2.71 \pm 1.33$ \\
\hline
\end{tabular}

${ }^{1}$ Encapsulation Efficiency

${ }^{2}$ Error expressed as standard deviation of three batches

${ }^{3}$ Polydispersity (PD) expressed as standard deviation of the size distribution, averaged across experimental repeats

Table 1: Characteristics of nanoparticles used in biodistribution and treatment studies

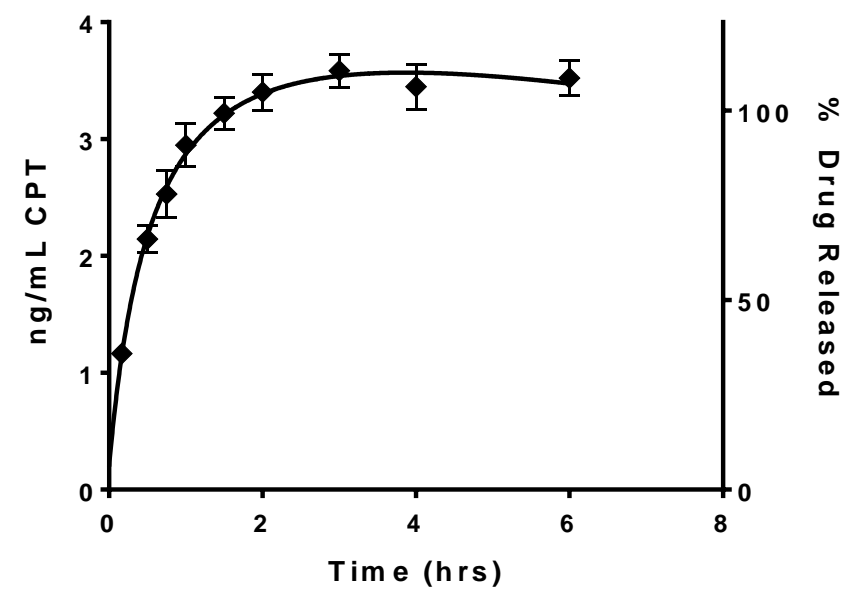

Figure 2: Camptothecin is released from RVG-PLGA-CPT nanoparticles within 3 hours. Error bars for individual time points represent standard deviation of three independent measurements.

To measure biodistribution of nanoparticle payload, we first analyzed the delivery of DiR from nanoparticles administered by injection into the lateral tail vein of healthy mice. DiR was detected two hours following intravenous administration in the kidneys, liver, lungs, heart, femur muscle, and uterine horns (Figure 3). The greatest amount of DiR was detected in the liver $(13.9 \mu \mathrm{g} / \mathrm{g}$ and $13.1 \mu \mathrm{g} / \mathrm{g}$ for biotin-PLGA-DiR and RVG-PLGA-DiR, respectively), followed by the lungs $(4.52 \mu \mathrm{g} / \mathrm{g}$ and $4.35 \mu \mathrm{g} / \mathrm{g}$, for biotin-PLGA-DiR and RVG-PLGA-DiR respectively). No significant differences in delivery were observed for RVG29- versus biotin-modified nanoparticles in any peripheral organs or blood plasma. DiR was detected readily in the brain at a concentration of $79.5 \mathrm{ng} / \mathrm{g}$ and $101 \mathrm{ng} / \mathrm{g}$ for biotin- versus RVG29-modified formulations, respectively. RVG29-modification of nanoparticles thus produced a $27 \%$ increase in delivery to the brain at two hours, which was significant ( $\mathrm{p}=0.041$, Student's 1-tailed ttest). In whole spinal cord, DiR was detected at $51.4 \mathrm{ng} / \mathrm{g}$ for both RVG29- and biotin-modified formulations. Thus, delivery to the spinal cord was lower than delivery to the brain, and no enhancement due to RVG29 was observed. To determine whether the brain targeting effects reflected some unique feature of the DiR payload, we also evaluated the brain-specific delivery of other encapsulated small molecules, including coumarin 6 and NR. These data demonstrated that the targeting effects were consistent, if not higher, for different payloads; significantly increased delivery (40-60\%) was observed 
in the brain two hours after injection for these agents in multiple experimental repeats (Supplementary Figure 1).

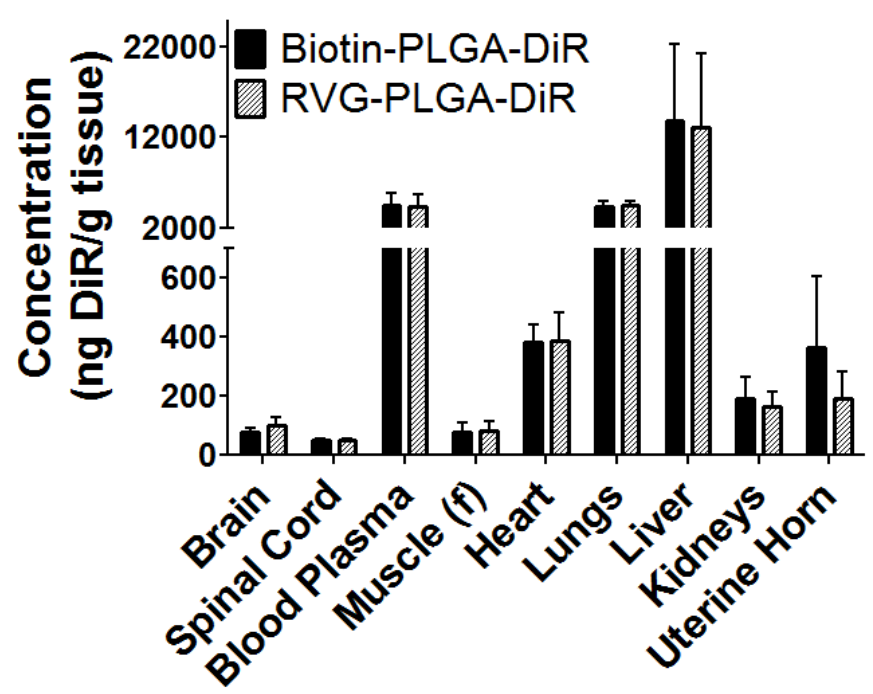

Figure 3: Biodistribution of Biotin-PLGA-DiR and RVG-PLGA-DiR nanoparticles in peripheral organs two hours post-injection. Error bars represent standard deviation with $n=6$ mice per group. $(f)=$ femur

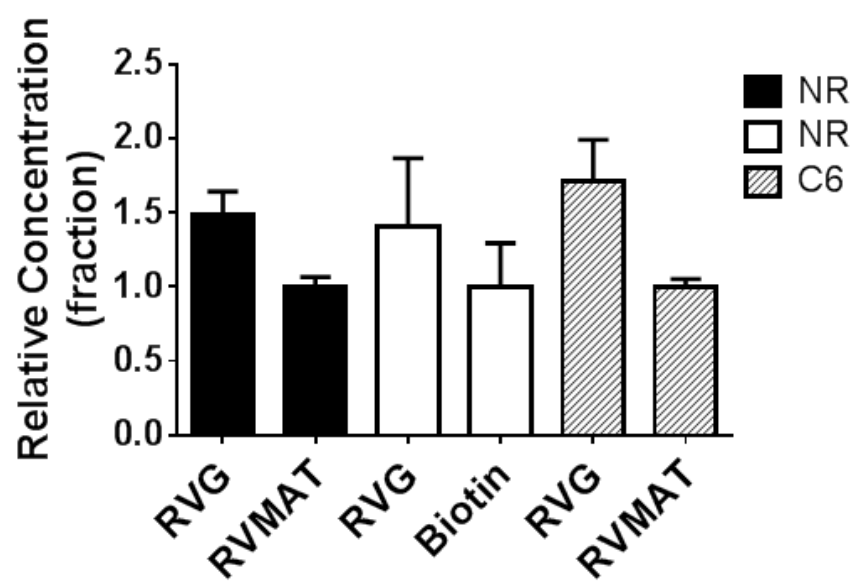

Supplementary Figure 1: Improved targeting of payload to the brain after two hours using RVG29-modified PLGA nanoparticles is consistent across different fluorescent encapsulated molecules. RV-MAT is a control peptide derived from the matrix protein of rabies virus, which has no known role in rabies virus neurotropism (sequence: N term-MNLLRKIVKNRRDEDTQKSSPASAPLDDG-C2-Biotin). These data demonstrate consistency of apparent targeting in experimental repeats, and also across both a biotin and an RV-MAT control. Each set of data represents an independent experiment, with $n=3-5$ mice per treatment group and delivered dose of 4-6mg PLGA per mouse. Ratios are normalized to RV-MAT or biotin control, as labeled for each data set. NR $=$ Nile red. $C 6=$ Coumarin 6

We were interested to determine whether there would be differences in the quantity of payload delivered to major anatomical regions within the brain and spinal cord (Figure 4 and Table 2). A two-way analysis of variance (ANOVA) identified significant effects of CNS region $(\mathrm{F}(10,90=21.19, \mathrm{p}<0.001)$ and significant interaction between CNS region and targeting $(\mathrm{F}(10,90)=2.39, \mathrm{p}=0.0145)$ on delivered payload concentration. The highest delivery was detected in the cortex for both RVG29- and biotinmodified formulations (123 versus $89.6 \mathrm{ng} / \mathrm{g}$, respectively), followed by the cerebellum ( 98.8 versus $79.9 \mathrm{ng} / \mathrm{g}$, respectively). We hypothesized that variations in payload delivery to different regions within the brain would be due to regional differences in blood volume; consistent with this hypothesis, the 
relationship between cerebral blood volume [31] and DiR delivered from nanoparticles was linear for both targeted and non-targeted formulations (Figure 5), with calculated Pearson correlation coefficients of 0.979 and 0.854 for biotin- and RVG29-modified nanoparticles ( $<<0.01$ for both). For the spinal cord, payload delivery tended to increase slightly in the caudal direction $(48.7,49.9,53.2$, and $55.1 \mathrm{ng} / \mathrm{g}$ in cervical, thoracic, lumbar, and sacral segments). Some evidence for apparent targeting (i.e., increased delivery for RVG29- versus biotin-modified formulations) was observed in the cervical region of the spinal cord (15\% increase for RVG29- versus biotin-modified nanoparticles), although mild decreases in delivery were observed for targeted formulations in thoracic, lumbar, and sacral regions. In contrast to the spinal cord, enhancements were observed consistently across all major brain regions for RVG29 modified nanoparticles, with each region displaying a higher concentration of DiR delivered from RVG29-modified nanoparticles than DiR from biotin-modified nanoparticles. The highest targeted delivery was observed in the cortex ( $36.8 \%$ increase) and cerebellum ( $23.6 \%$ increase), followed closely by the midbrain ( $22.4 \%$ increase) and striatum (21.4\% increase). There was no relationship between targeting ratio by brain region and cerebral blood volume, which had a Pearson correlation coefficient of 0.246 (data not shown). For the RVG29-modified group, delivery was significantly lower to the striatum than many other brain regions, including cortex, midbrain, cerebellum, and brainstem (Figure 4A, $\mathrm{p}<0.001,0.01,0.001,0.05$, respectively, Bonferroni posttest).

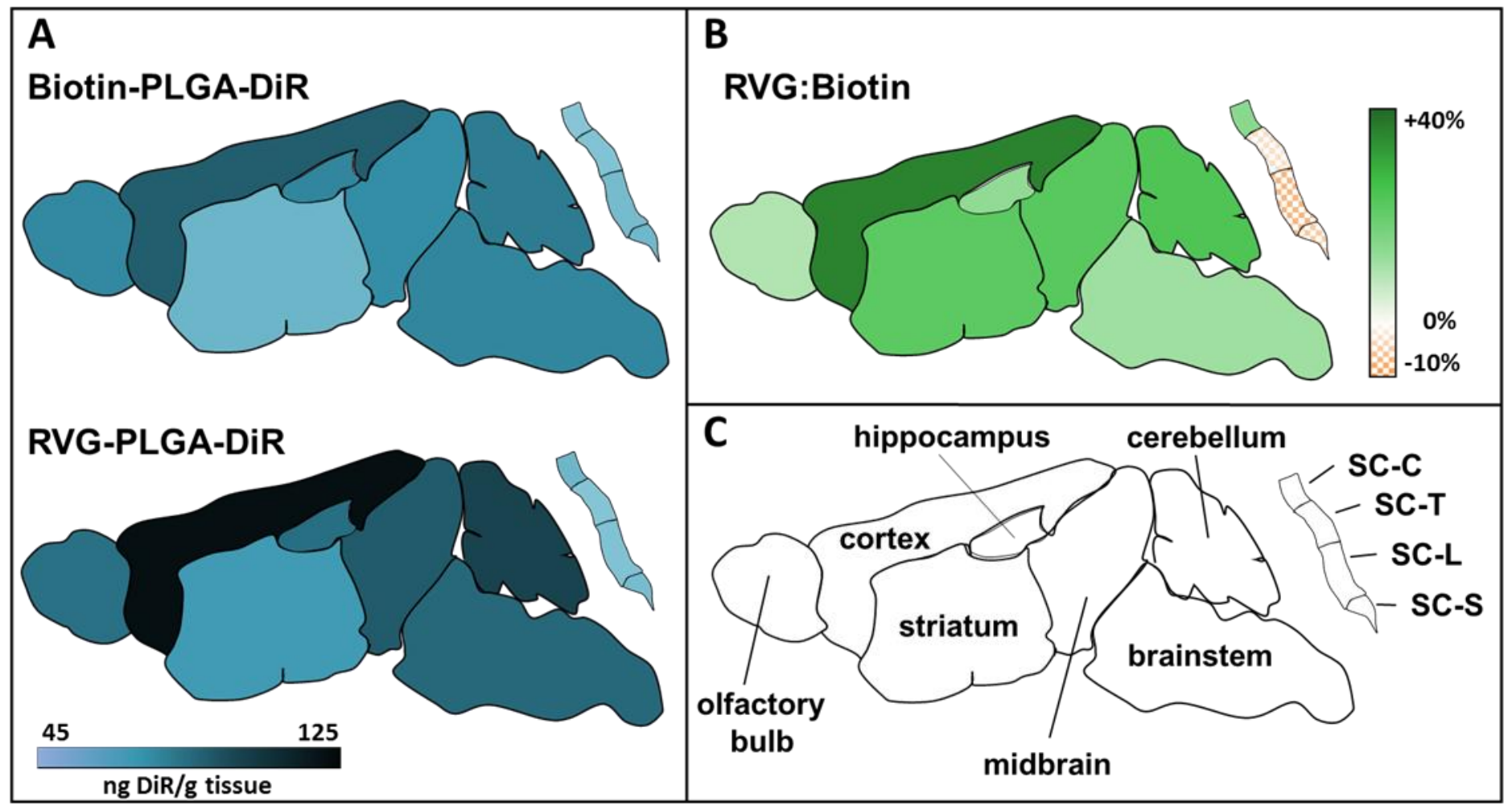

Figure 4: The distribution of targeted and non-targeted DiR nanoparticles varies by brain region (these data are a heatmap depiction of the data provided in Table 2). (A) Average total payload delivered to each brain and spinal cord region ( $n g$ DiR/g tissue) ( $n=6$ per group). (B) Targeting effect by region, as measured by the ratio of RVG-PLGA-DiR to Biotin-PLGA-DiR. (C) Map of brain and spinal cord regions, sagittal view. SC=Spinal Cord; $C=$ Cervical, $T=$ Thoracic, $L=$ Lumbar, $S=$ Sacral. 


\begin{tabular}{|lccc|}
\hline Region & Biotin $^{\mathbf{1}}(\mathbf{n g} / \mathbf{g})$ & RVG $^{\mathbf{1}}(\mathbf{n g} / \mathbf{g})$ & Targeting $^{\mathbf{2}}$ \\
\hline Whole Brain & $79.5 \pm 10.8$ & $101 \pm 25.1$ & $27.0 \%$ \\
\hline Striatum & $55.9 \pm 10.7$ & $67.9 \pm 27.0$ & $21.4 \%$ \\
\hline Midbrain & $73.9 \pm 22.3$ & $90.4 \pm 25.8$ & $22.4 \%$ \\
\hline Olfactory Bulb & $76.4 \pm 13.0$ & $83.2 \pm 23.2$ & $8.94 \%$ \\
\hline Brainstem & $76.9 \pm 17.3$ & $85.6 \pm 14.5$ & $11.3 \%$ \\
\hline Cortex & $89.6 \pm 16.7$ & $123 \pm 36.4$ & $36.8 \%$ \\
\hline Hippocampus & $62.9 \pm 5.74$ & $71.1 \pm 16.2$ & $13.0 \%$ \\
\hline Cerebellum & $79.9 \pm 15.3$ & $98.8 \pm 13.1$ & $23.6 \%$ \\
\hline Whole Spinal Cord & $39.6 \pm 4.18$ & $51.4 \pm 2.81$ & $0.04 \%$ \\
\hline SC-C & $48.7 \pm 8.69$ & $55.8 \pm 6.34$ & $14.5 \%$ \\
\hline SC-T & $49.9 \pm 3.89$ & $48.5 \pm 2.86$ & $-2.74 \%$ \\
\hline SC-L & $53.2 \pm 8.24$ & $49.5 \pm 5.89$ & $-6.86 \%$ \\
\hline SC-S & $55.1 \pm 2.07$ & $52.4 \pm 6.20$ & $-4.93 \%$ \\
\hline
\end{tabular}

${ }^{1}$ Average $\pm \mathrm{SD}, \mathrm{n}=6$ mice per group

${ }^{2}$ Targeting is defined as the percent increase or decrease in RVG average relative to biotin

${ }^{3} \mathrm{SC}=$ Spinal Cord; $\mathrm{C}=$ Cervical, $\mathrm{T}=$ Thoracic, $\mathrm{L}=$ Lumbar, $\mathrm{S}=$ Sacral.

Table 2: Delivery of DiR from RVG29- and biotin-modified nanoparticles, measured within specific regions of the CNS
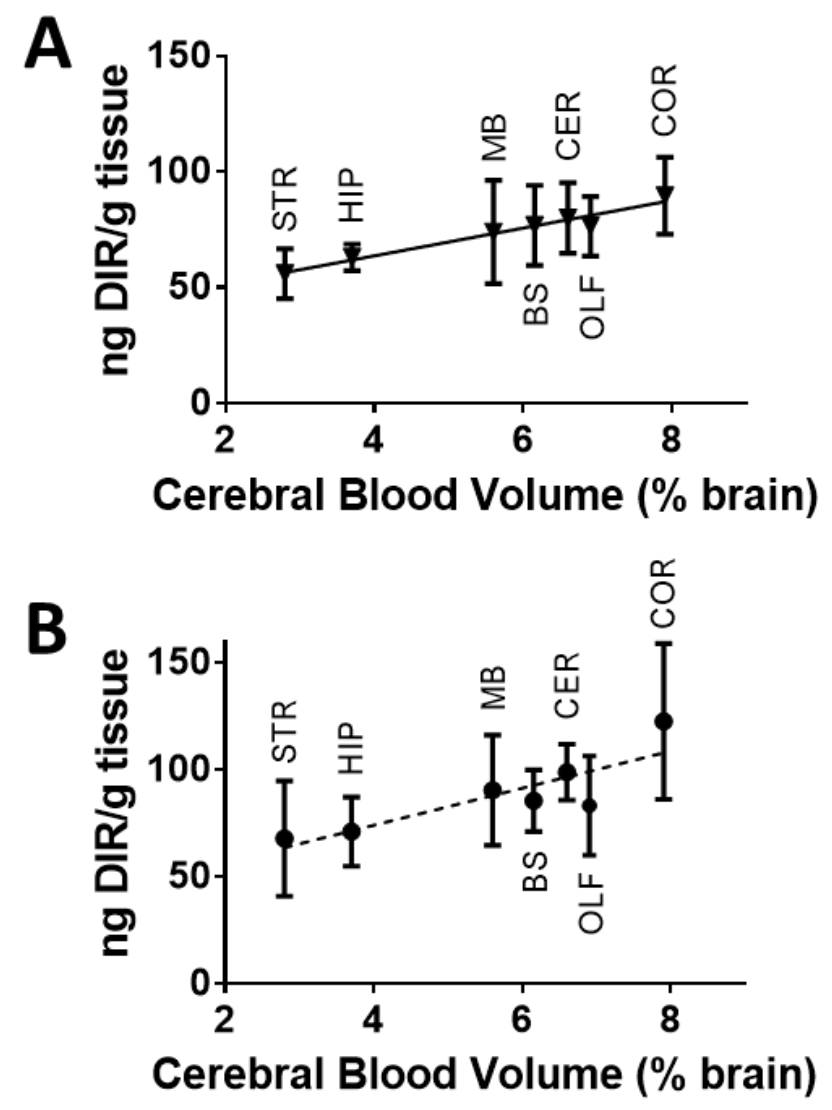

Figure 5: Regional blood volume correlates with payload distribution in the brain delivered by (A) RVG-PLGADiR and (B) Biotin-PLGA-DIR. Cerebral blood volume represents percent volume of blood per volume of brain 
tissue, obtained from published data for the mouse [31]. STR=Striatum, HIP=Hippocampus, MB=Midbrain, $B S=$ Brainstem, $C E R=$ Cerebellum, OLF=Olfactory Bulb, COR=Cortex.

Treatment of Intracranial Tumors with Targeted Nanoparticles

The next series of experiments were designed to test whether enhanced delivery via RVG29 targeting would enhance the efficacy of the encapsulated chemotherapeutic camptothecin (CPT). Syngeneic GL261-Luc2 tumors were induced in the striatum of immune competent mice. Saline, RVG-PLGA (drug empty nanoparticles), Biotin-PLGA-CPT, or RVG-PLGA-CPT were administered on a weekly basis to match a $12 \mathrm{mg} / \mathrm{kg}$ CPT dose by injection into the lateral tail vein beginning 8 days after tumor induction. Consistent with prior work [8], encapsulated CPT was effective at slowing tumor growth compared to saline injected controls (Figure 6A and 6C). No differences in tumor growth were detected between RVG29 and biotin nanoparticle modifications. Median survival for saline, RVG-PLGA, Biotin-PLGA-CPT, and RVG-PLGA-CPT was 16.5, 19, 27, and 23 days, respectively (Figure 6B). Survival was significantly prolonged for Biotin-PLGA-CPT and RVG-PLGA-CPT treated subjects relative to either saline or RVG-PLGA controls ( $\mathrm{p}<0.05$ for each comparison), although RVG-PLGACPT did not prolong survival in comparison to Biotin-PLGA-CPT. Taken as a whole, these data demonstrate that encapsulated CPT slowed tumor growth, resulting in prolonged survival, but targeting did not enhance treatment efficacy.
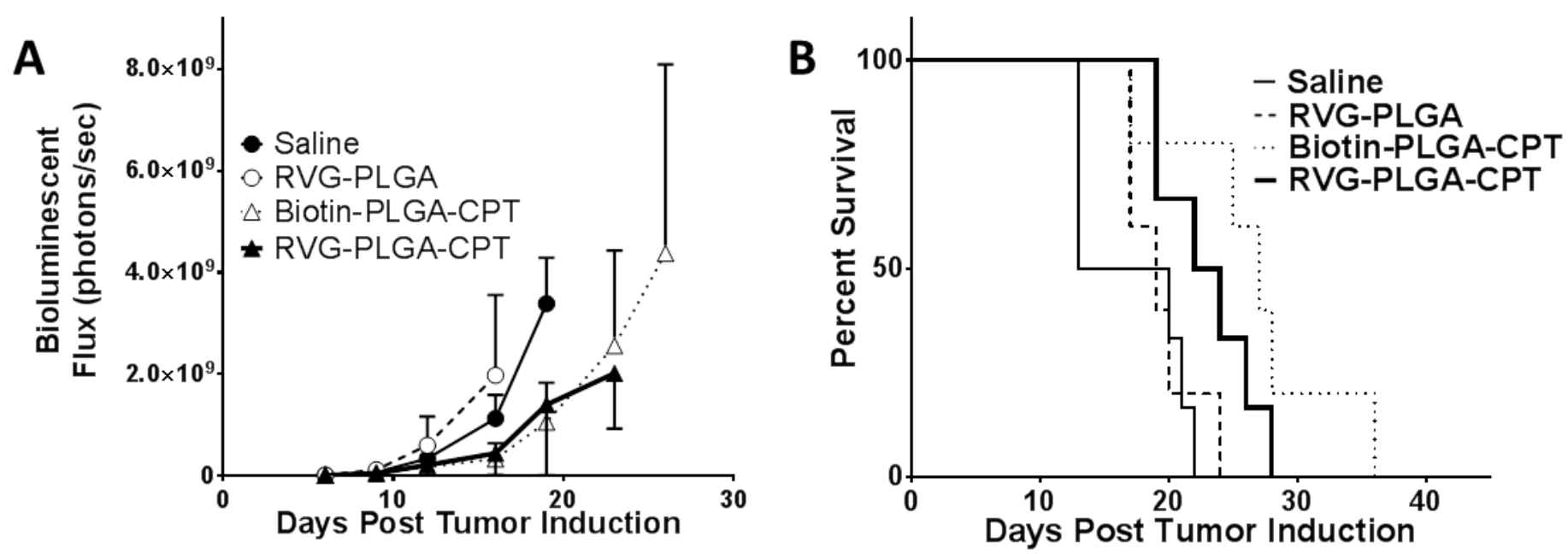

\begin{tabular}{|cccc|}
\hline C & $\begin{array}{c}\text { Subjects } \\
\text { (n) }\end{array}$ & $\begin{array}{c}\text { Tumor Doubling Time } \\
\text { (days [95\% CI]) }\end{array}$ & $\begin{array}{c}\text { Median Survival } \\
\text { (days) }\end{array}$ \\
\hline Saline & 6 & $2.46[2.41,2.52]$ & 16.5 \\
\hline RVG-PLGA & 5 & $2.50[2.38,2.64]$ & 19 \\
\hline Biotin-PLGA-CPT & 5 & $3.14[3.01,3.29]$ & 27 \\
\hline RVG-PLGA-CPT & 6 & $3.15[2.99,3.34]$ & 23 \\
\hline
\end{tabular}

Figure 6: PLGA nanoparticles encapsulating camptothecin slow tumor growth and prolong survival in vivo. (A) Normalized growth of GL261-Luc2 brain tumors monitored by IVIS, represented in photons/second. (B) KaplanMeier survival curve with $n=5-6$ per group. (C) Summary of treatment study results. The $95 \%$ confidence intervals for tumor doubling time are shown in brackets. 
The biodistribution experiments suggested a consistent enhancement in delivery of small molecules from RVG29-modified nanoparticles 2 hours after injection. However, when we examined the biodistribution of DiR or NR loaded nanoparticles 6 hours after administration (Figure 7), the presence of the targeting ligand did not affect or even tended to reduce whole brain delivery of encapsulated payloads (targeting ratio, RVG29 average divided by biotin average, of 0.98 and 0.93 for NR and DiR experiments, respectively). The apparent targeting measurements were therefore reproducible at two hours and generalizable to at least three encapsulated small molecules, but targeting was not prolonged (i.e., no enhancements in delivery were observed after six hours). To test the aqueous stability of RVG29, mice were injected with nanoparticles that had been pre-incubated in saline at $37^{\circ} \mathrm{C}$ for four hours (Figure 7). No apparent targeting was observed two hours after injection of these pre-incubated nanoparticles (targeting ratio, RVG29 average divided by biotin average, of 0.96). To test whether aggregation accounted for this loss of targeting, a sample of nanoparticles was maintained at $37^{\circ} \mathrm{C}$ for 6 hours; DLS measurement confirmed that the average hydrodynamic diameter did not increase over time (Supplementary Figure 2). We expect that additional loss in targeting may occur in vivo via formation of a protein corona on the outer surface of the nanoparticle [34], although this was not investigated since incubation in saline was sufficient to remove the targeting ability of RVG29-modified nanoparticles.

In the process of debugging the lost targeting, we conducted kinetic experiments that measured the delivery of different payloads over multiple time points. These experiments demonstrated that NR and DiR produce opposite interpretations of apparent nanoparticle delivery. For NR-loaded nanoparticles, we observed rapid clearance of NR from the brain, whereas, for DiR-loaded nanoparticles, we observed an apparent accumulation of DiR in the brain over time (Figure 8a). When free DiR was administered by tail vein injection, the brain signal also increased over time (Figure 8b), with free DiR accounting for up to $38 \%$ and $40 \%$ of the signal at 30 minutes and 6 hours, respectively. To our knowledge, brain measurements following an injection of free DiR have not been reported in literature. Brain accumulation of DiR following administration as a bolus intravenous injection was not expected and is not typical kinetic behavior for a small molecule. These data demonstrate that an increase in DiR signal over time does not necessarily reflect accumulation of the nanocarrier itself, and instead could be accounted for by the behavior of free (non-encapsulated) DiR.

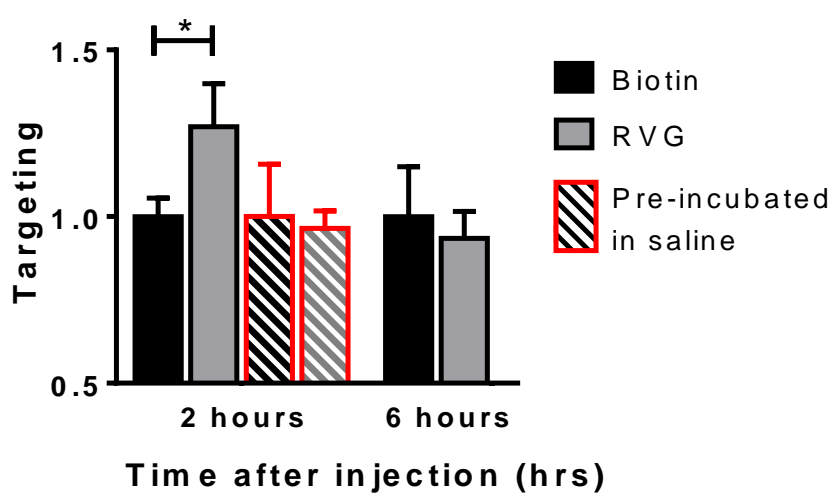

Figure 7: Loss of apparent targeting is observed after two hours in solution or in circulation. $R V G-$ PLGA-DiR or Biotin-PLGA-DiR was injected via tail vein immediately after resuspension and measured in the brain after two or four hours in circulation (solid bars). In a separate group of mice, RVG-PLGADiR or Biotin-PLGA-DiR was resuspended in saline and incubated at $37^{\circ} \mathrm{C}$ for four hours, then injected into the tail vein and measured two hours later, for a total of six hours in solution (striped bars). Ratios are group averages normalized to biotin control for each condition measured, with *p<0.05. The two hour biotin/RVG pair is also displayed in Figure 3. 


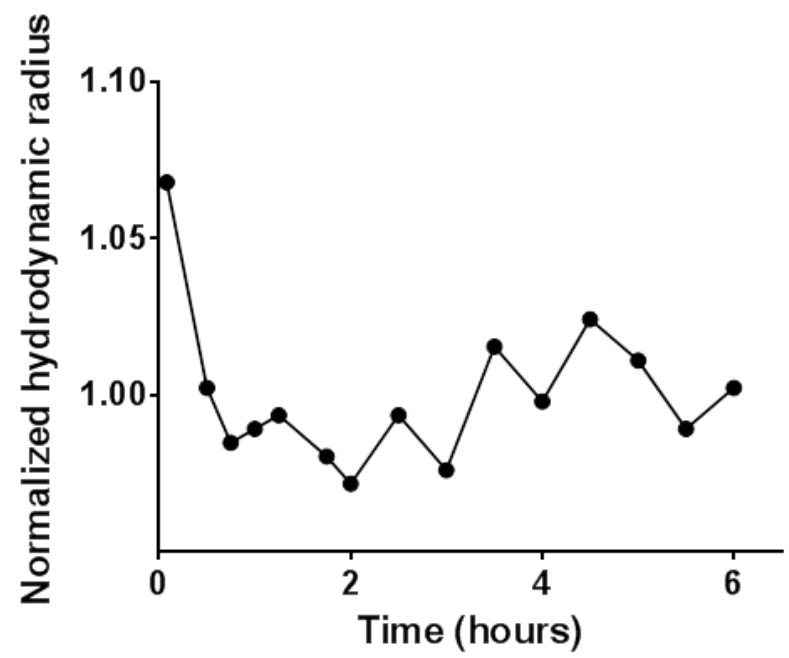

Supplementary Figure 2: The hydrodynamic radius of $R V G-P L G A-D i R$ does not change over a period of six hours for nanoparticles maintained in aqueous conditions at $37^{\circ} \mathrm{C}$.

A

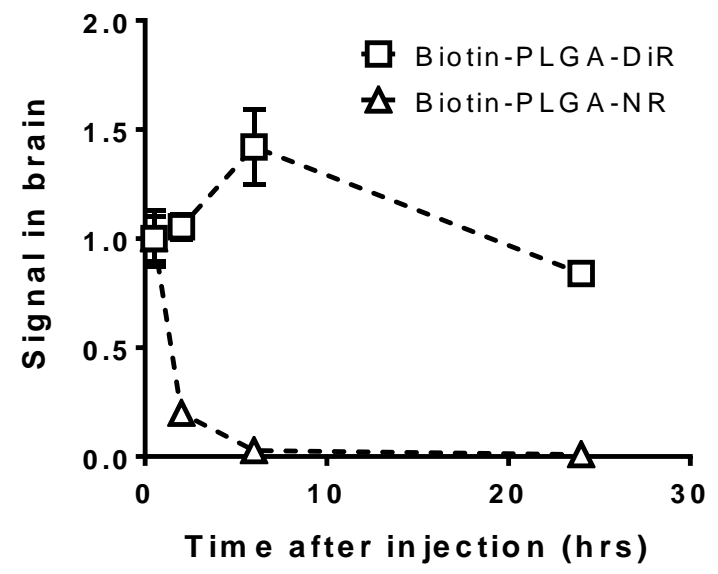

B

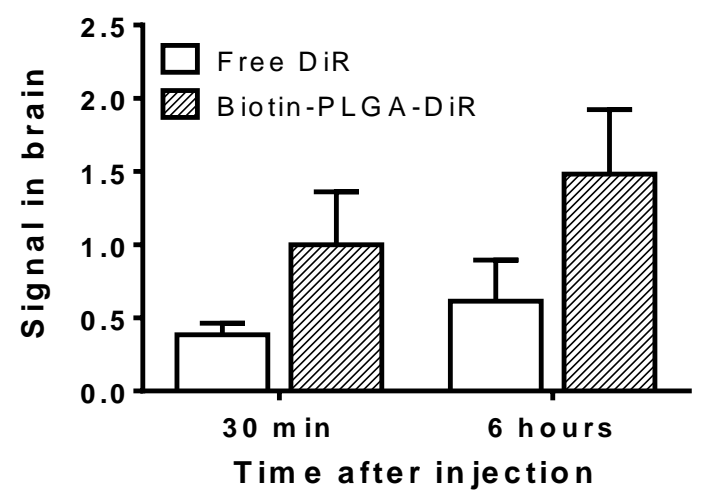

Figure 8: Different nanoparticle payloads yield different apparent brain delivery kinetics. (A)NR or DiR loaded nanoparticles were administered to healthy mice and fluorescent signal was measured in brain homogenate. $(B)$ When free DiR is administered to healthy mice, accumulation is observed in the brain, which accounts for $\sim 40 \%$ of the signal detected for mice provided with an equivalent dose of DiR loaded nanoparticles. The same data are represented in each panel for the Biotin-PLGA-DiR group. Plots 
shown mean with standard error with 6 mice/group for Biotin-PLGA-DiR and 3 mice/group for all other conditions.

\section{DISCUSSION}

These studies are some of the first to measure quantitative differences in small molecule delivery to different tissue sites within the CNS for intravenously administered nanoparticles. Calvo and colleagues tracked the distribution of ${ }^{14} \mathrm{C}$-labeled, PEGylated polycyanoacrylated nanoparticles and identified significant within-CNS regional differences in delivery that would be expected to influence drug function [35]. In our experiments, nanoparticle-mediated delivery of encapsulated DiR from nanoparticles was also not uniform. For both targeted and non-targeted formulations, brain delivery was directly correlated to regional cerebral blood volume. Delivery magnitude to different regions varied by as much as $60-80 \%$ within the brain, and by as much as $150 \%$ across the entire CNS (e.g., cortex versus thoracic spinal cord). These differences suggest that the targeting ability of a nanoparticle would need to be high to overcome differences in small molecule exposure to specific brain regions as governed by blood flow. In prior work, we observed a 13 -fold increase in DiR delivery from nanoparticles to the core of late-stage intracranial GL261 versus contralateral (healthy) brain.[8] Although EPR gains are expected to depend on tumor type, stage, and carrier system [9] enhanced delivery to tumor core could easily dominate the relatively smaller gains that are typically achieved for targeted delivery to healthy parenchyma. To treat invading cells in GBM, delivery improvements across an intact BBB would need to be high. In this work, we observed that delivery to the striatum, where tumors were induced, was significantly lower than other brain regions, including other common sites for brain tumors, such as cortex, cerebellum, and brainstem. Further, we observed that apparent targeting to the cortex (which represented $44 \%$ of the total brain mass) was twice as high as the brain averages with cortex removed. High cortex values drive whole brain homogenate values, thus potentially misrepresenting the delivery of brain available agents that would occur for any disease not occurring in the cortex. Although the delivery of DiR would not be expected to represent the spatial pattern of delivery for all encapsulated molecules, these studies highlight the importance of considering CNS region in the development of new small molecule delivery strategies. It will be an interesting subject of future work to determine whether engineering strategies that enhance nanoparticle or drug interaction with defined regions of brain or brain vasculature [36] would improve exposure of circulating agents to specific CNS regions.

Nanoparticles have been studied extensively for their ability to deliver encapsulated agents selectively to the injured or diseased spinal cord, such as in neurodegeneration or inflammation [37-40]. By comparison, relatively little is known about the features that govern delivery of encapsulated agents from nanoparticles to intact spinal cord. The blood spinal cord barrier (BSCB) is often thought of as a physiological extension of the BBB, possessing non-fenestrated capillary endothelium, tight junctions, and efflux pumps. However, morphological and functional characteristics of barrier endothelial cells vary by location and are distinct in the spine relative to the brain [41]. Given that the rabies virus itself possesses tropism for motor neurons, we expected to observe targeting in the spinal cord. Nanoparticles delivered payload to spinal cord, however, no apparent enhancement in delivery from RVG29 was observed in the spinal cord. $\mathrm{GABA}_{\mathrm{B}}$ receptors, a class of proteins that were recently described as a potential binding partner for RVG29[25], are present both on neurons and on the brain capillary endothelial cells that compose the BBB. Interestingly, relative to other brain regions, $G_{A B} A_{B} R$ is reported to be highly enriched in the cortex and present in much lower quantities in hippocampus, bulk striatum, and spinal cord[42], which mirrors the patterns of apparent delivery enhancements observed for RVG29-modified nanoparticles. Our data support a model of "targeting" whereby RVG29 modification of PLGA nanoparticles transiently enhances their affinity for the surface of brain endothelial cells via interaction with $\mathrm{GABA}_{\mathrm{B}}$ Rs. The fact that the enhancement in delivery observed for 
RVG29-modified nanoparticles is not maintained at later time points due to aqueous instability of the ligand further supports a non-internalization model of this apparent targeting. The association of RVG29-modified nanoparticles with the cell surface appears to be temporary.

Prior studies demonstrated the ability of RVG29 to improve the CNS efficacy of encapsulated or tethered nucleic acids and proteins [12, 14-25]. In contrast, evidence for functional enhancement of small molecules via RVG29 remains minimal. Enhanced in vitro and brain uptake of the small molecule antifungal agent itraconazole was observed for RVG29-modified albumin nanoparticles [43], although this study did not evaluate therapeutic potency of the targeted formulation in vivo. Thus, the initial motivation for the studies described here was to determine whether RVG29 modification of nanoparticles would improve therapeutic efficacy of the chemotherapeutic agent CPT. CPT is a topoisomerase I inhibitor that is effective at killing cancer cells but is also hydrophobic, poorly bioavailable, and highly toxic [44]. The opportunity for improved therapeutic efficacy of CPT rests either on improving its tolerability, which would enable a higher dose, or improving its delivery, which would increase potency in target tissue. To deliver drug to tumor cells that do not reside in the leaky tumor core, nanoparticles would need to engage in prolonged interaction (either by uptake or surface residence) with cells to be effective. In accordance with prior work, we achieved a high loading of CPT in PLGA, which was sufficient to slow the growth of intracranial GL261 tumors when nanoparticles were administered systemically [8]. However, we did not observe any evidence that RVG29 enhanced CPT efficacy. There were no differences in either median survival or tumor growth between the targeted and non-targeted group. Although it is possible that small differences in tumor growth are masked by variability inherent in bioluminescent measurement, any such differences did not ultimately affect overall survival. Further analysis leads us to two important points of discussion: first, a critical examination of common methods used for evaluating new nanoparticle designs, and, second, insight into the mechanisms by which brain-specific delivery of encapsulated small molecules appears to be achieved.

The most direct method for measuring the delivery of drugs from nanocarriers would be pharmacokinetic analysis of actual drug levels in tissue. Alternatively, fluorescent or radioactive labeling strategies can permit direct tracking of the nanoparticle itself [45, 46]. Evaluation of new nanoparticle designs would ideally incorporate both measurements, since the techniques provide complementary information. However, these methods are not widely applied in tandem due to several important drawbacks. Both strategies require careful optimization of experimental approaches that are specific to drug or carrier. In the case of the direct labeling strategy, it is extremely important to validate that the label itself is stably attached to the carrier in a manner that does not alter the biophysical properties of the carrier. As an alternative to these expensive and difficult to implement strategies, nanocarrier vehicles are commonly loaded with small, fluorescent drug surrogates. Measurement of biodistribution is a comparatively simple technique that proceeds with one of two expectations: either, that the freely loaded small molecule is well-retained within the carrier, or, if it exits the nanoparticle, that its movement in tissue might be considered sufficiently typical of the drug of interest, possessing similar molecular weight or charge. Our data suggest that, in fact, these small molecule surrogates are not retained within polymer nanoparticles in vivo, and that their rate of delivery to tissue largely reflects their own ability move through biological environments. DiR, for example, experiences a highly deceptive accumulation in the brain, even in its free form. DiR and other carbocyanine dyes are commonly used to track delivery of nanoparticle formulations. Yet, their retention within or release from a nanocarrier would be expected to be highly specific to both the carrier and the lipid composition of its biological environment; the specific experimental approach of using carbocyanine dyes for biodistribution may need to be revisited. We suggest that certain biodistribution experiments may be useful to provide an indication of relative differences in nanoparticle residence time within single tissues 
of interest. However, it is clear that the distribution of a drug surrogate should only ever be interpreted in context with other measures of delivery.

We propose that different kinetics measured by different payloads can be explained by differences in the ability of molecules to transfer to and from biological membranes. NR is known to experience rapid cell to cell membrane transfer [47] whereas, as a carbocyanine family member, DiR is expected to be wellretained within lipid compartments [48]. Thus, NR delivered to cells would rapidly track the washout of nanoparticles from blood, whereas DiR would be relatively immobilized in cellular membranes, egressing slowly into the lipid rich environment of the brain. It has been proposed that small molecule delivery from nanocarriers is primarily mediated by direct transfer of payload from nanoparticle to cell in vitro, where the majority of small molecule delivery does not rely on active uptake of the nanoparticle $[47,49]$. Our in vivo data support this model, and suggest that apparent brain delivery may in fact reflect peripheral circulation of nanoparticles, either via direct transfer of encapsulated agent from nanoparticle to $\mathrm{BBB}$ cells or by enhancing drug source in the periphery.

Release of the active agent is an additional confounder that should be considered when using biodistribution measurements to interpret drug delivery [50]. For example, some molecules could release very quickly, before targeting effects become apparent, whereas others may be better retained, which could over-represent targeting as it would be expected for the actual drug. Ultimately, the release of an encapsulated molecule from its nanocarrier will depend on properties that are specific to the molecule, carrier, and its particular in vivo environment. Since in vivo measurement of drug release is not straightforward and cannot be directly predicted from in vitro data, this further complicates the use of drug surrogates in making decisions about nanocarrier design. As our data demonstrates, unexpected peculiarities of certain molecules could easily mislead (e.g., as evident in the brain accumulation of DiR). It is important to note that encapsulation of payload within a nanoparticle still provides drug delivery benefits that should be considered in the design of the nanoparticle (e.g., reduced toxicity, prolonged circulation time, etc.). This highlights the need for improved drug/drug surrogate retention in designing targeted formulations: slower release from long-circulating nanoparticle formulations will reduce drug clearance to enhance delivery in target tissue, whether this occurs by extracellular transfer or actual uptake of the carrier.

Targeted drug delivery to the brain has not yet achieved widespread success. Our results support a model of nanoparticle-mediated brain delivery whereby molecules are transferred non-specifically from nanocarriers to the brain in absence of significant internalization of the nanoparticle.[47, 49] We suggest that RVG29 enhances delivery of small molecules from PLGA nanoparticles by producing a transient increase in affinity of the nanoparticle for the surface of the BBB. It is possible that further engineering of the nanoparticle (e.g., different modification density, alterations to the peptide structure) could alter ligand functionality. By bringing nanoparticles in closer proximity to endothelial cells, payload gains a better ability to transfer directly into cells. Delivery is thus fundamentally driven by the particle to cell transfer and clearance processes that are unique to each small molecule payload of interest. These observations emphasize the challenge and importance of achieving substantial internalization of PLGA nanoparticles in target cells in vivo and also highlight major experimental considerations for evaluation of targeted drug carriers in tissue. We focused here on confounders in the analysis of PLGA nanoparticle biodistribution. However, these concerns may also apply to carriers (liposomes, micelles, etc.) where the drug surrogate being tracked is not covalently linked to the carrier and retention of the surrogate in the carrier has not been demonstrated in target tissue. We argue that functional evaluation of drug efficacy in the appropriate disease model would be best initiated at early stages of nanoparticle engineering.

\section{ACKNOWLEDGEMENTS}


The authors gratefully acknowledge funding from the Ben and Catherine Ivy Foundation, the Barrow Neurological Foundation, the Department of Defense (W81XWH-14-1-0311), the National Science Foundation (DGE-1311230) and the National Institutes of Health (R01-CA149128, T32-DA022975). We also thank Dr. Adrienne C. Scheck at Barrow Neurological Institute for providing GL261-Luc2 cells.

1. Patel, T.R., Nanocarrier-based therapies for CNS tumors. CNS oncology, 2014. 3(2): p. 115-22.

2. Li, M., et al., Functional nanoparticles in targeting glioma diagnosis and therapies. Journal of nanoscience and nanotechnology, 2014. 14(1): p. 415-32.

3. Tortorella, S. and T.C. Karagiannis, The significance of transferrin receptors in oncology: the development of functional nano-based drug delivery systems. Current drug delivery, 2014. 11(4): p. 427-43.

4. van der Meel, R., et al., Ligand-targeted particulate nanomedicines undergoing clinical evaluation: current status. Advanced drug delivery reviews, 2013. 65(10): p. 1284-98.

5. Kreuter, J., Drug delivery to the central nervous system by polymeric nanoparticles: what do we know? Advanced drug delivery reviews, 2014. 71: p. 2-14.

6. Alam, M.I., et al., Strategy for effective brain drug delivery. European journal of pharmaceutical sciences : official journal of the European Federation for Pharmaceutical Sciences, 2010. 40(5): p. 385-403.

7. Prabhakar, U., et al., Challenges and key considerations of the enhanced permeability and retention effect for nanomedicine drug delivery in oncology. Cancer Research, 2013. 73(8): p. 2412-7.

8. Householder, K.T., et al., Intravenous delivery of camptothecin-loaded PLGA nanoparticles for the treatment of intracranial glioma. International journal of pharmaceutics, 2015. 479(2): p. 374-80.

9. Bertrand, N., et al., Cancer nanotechnology: the impact of passive and active targeting in the era of modern cancer biology. Advanced drug delivery reviews, 2014. 66: p. 2-25.

10. Langevin, C., et al., Rabies virus glycoprotein (RVG) is a trimeric ligand for the N-terminal cysteine-rich domain of the mammalian p75 neurotrophin receptor. The Journal of biological chemistry, 2002. 277(40): p. 37655-62.

11. Lafon, M., Rabies virus receptors. Journal of neurovirology, 2005. 11(1): p. 82-7.

12. Kumar, P., et al., Transvascular delivery of small interfering RNA to the central nervous system. Nature, 2007. 448(7149): p. 39-43.

13. Liu, Y., et al., Brain-targeting gene delivery and cellular internalization mechanisms for modified rabies virus glycoprotein RVG29 nanoparticles. Biomaterials, 2009. 30(25): p. 4195-202.

14. Son, S., et al., RVG peptide tethered bioreducible polyethylenimine for gene delivery to brain. Journal of Controlled Release, 2011. 155(1): p. 18-25.

15. Hwang do, W., et al., A brain-targeted rabies virus glycoprotein-disulfide linked PEI nanocarrier for delivery of neurogenic microRNA. Biomaterials, 2011. 32(21): p. 4968-75.

16. Liu, Y., et al., Targeting caspase-3 as dual therapeutic benefits by RNAi facilitating brain-targeted nanoparticles in a rat model of Parkinson's disease. PloS one, 2013. 8(5): p. e62905.

17. Zadran, S., et al., RVG-mediated calpain2 gene silencing in the brain impairs learning and memory. Neuromolecular medicine, 2013. 15(1): p. 74-81.

18. Kim, J.Y., et al., Brain-targeted delivery of protein using chitosan- and RVG peptide-conjugated, pluronicbased nano-carrier. Biomaterials, 2013. 34(4): p. 1170-1178.

19. Liu, Y., et al., Targeted imaging of activated caspase-3 in the central nervous system by a dual functional nano-device. Journal of Controlled Release, 2012. 163(2): p. 203-210.

20. Tao, Y., J. Han, and H. Dou, Brain-targeting gene delivery using a rabies virus glycoprotein peptide modulated hollow liposome: Bio-behavioral study. Journal of Materials Chemistry, 2012. 22(23): p. 11808-11815.

21. Alvarez-Erviti, L., et al., Delivery of siRNA to the mouse brain by systemic injection of targeted exosomes. Nature biotechnology, 2011. 29(4): p. 341-5.

22. Xiang, L., et al., Targeted delivery of large fusion protein into hippocampal neurons by systemic administration. Journal of drug targeting, 2011. 19(8): p. 632-636.

23. Fu, A., et al., Targeted delivery of proteins into the central nervous system mediated by rabies virus glycoprotein-derived peptide. Pharmaceutical research, 2012. 29(6): p. 1562-9. 
24. Chen, W., et al., Targeted brain delivery of itraconazole via RVG29 anchored nanoparticles. Journal of drug targeting, 2011. 19(3): p. 228-234.

25. Liu, Y., et al., Brain-targeting gene delivery and cellular internalization mechanisms for modified rabies virus glycoprotein RVG29 nanoparticles. Biomaterials, 2009. 30(25): p. 4195-4202.

26. McCall, R.L. and R.W. Sirianni, PLGA nanoparticles formed by single- or double-emulsion with vitamin ETPGS. Journal of visualized experiments : JoVE, 2013(82): p. 51015.

27. Fahmy, T.M., et al., Surface modification of biodegradable polyesters with fatty acid conjugates for improved drug targeting. Biomaterials, 2005. 26(28): p. 5727-36.

28. McCarron, P.A., et al., Antibody targeting of camptothecin-loaded PLGA nanoparticles to tumor cells. Bioconjugate chemistry, 2008. 19(8): p. 1561-9.

29. Dora, C.L., et al., Evaluation of antimetastatic activity and systemic toxicity of camptothecin-loaded microspheres in mice injected with B16-F10 melanoma cells. Journal of pharmacy \& pharmaceutical sciences : a publication of the Canadian Society for Pharmaceutical Sciences, Societe canadienne des sciences pharmaceutiques, 2006. 9(1): p. 22-31.

30. Meikle, A.D. and A.H. Martin, A rapid method for removal of the spinal cord. Stain technology, 1981. 56(4): p. 235-7.

31. Chugh, B.P., et al., Measurement of cerebral blood volume in mouse brain regions using micro-computed tomography. Neurolmage, 2009. 47(4): p. 1312-8.

32. Jacobs, V.L., et al., Current review of in vivo GBM rodent models: emphasis on the CNS-1 tumour model. ASN neuro, 2011. 3(3): p. e00063.

33. Abdelwahab, M.G., et al., Intracranial implantation with subsequent $3 D$ in vivo bioluminescent imaging of murine gliomas. Journal of visualized experiments : JoVE, 2011(57): p. e3403.

34. Sempf, K., et al., Adsorption of plasma proteins on uncoated PLGA nanoparticles. European journal of pharmaceutics and biopharmaceutics : official journal of Arbeitsgemeinschaft fur Pharmazeutische Verfahrenstechnik e.V, 2013. 85(1): p. 53-60.

35. Calvo, P., et al., Quantification and localization of PEGylated polycyanoacrylate nanoparticles in brain and spinal cord during experimental allergic encephalomyelitis in the rat. The European journal of neuroscience, 2002. 15(8): p. 1317-26.

36. McCall, R.L., et al., Pathogen-inspired drug delivery to the central nervous system. Tissue barriers, 2014. 2(4): p. e944449.

37. Saxena, T., et al., Nanocarrier-mediated inhibition of macrophage migration inhibitory factor attenuates secondary injury after spinal cord injury. ACS nano, 2015. 9(2): p. 1492-505.

38. Wang, Y.C., et al., Sustained intraspinal delivery of neurotrophic factor encapsulated in biodegradable nanoparticles following contusive spinal cord injury. Biomaterials, 2008. 29(34): p. 4546-53.

39. Chvatal, S.A., et al., Spatial distribution and acute anti-inflammatory effects of Methylprednisolone after sustained local delivery to the contused spinal cord. Biomaterials, 2008. 29(12): p. 1967-75.

40. Gaudin, A., et al., Squalenoyl adenosine nanoparticles provide neuroprotection after stroke and spinal cord injury. Nature nanotechnology, 2014. 9(12): p. 1054-1062.

41. Bartanusz, V., et al., The blood-spinal cord barrier: morphology and clinical implications. Annals of neurology, 2011. 70(2): p. 194-206.

42. Bowery, N.G., A.L. Hudson, and G.W. Price, GABAA and GABAB receptor site distribution in the rat central nervous system. Neuroscience, 1987. 20(2): p. 365-83.

43. Chen, W., et al., Targeted brain delivery of itraconazole via RVG29 anchored nanoparticles. Journal of drug targeting, 2011. 19(3): p. 228-34.

44. Ulukan, H. and P.W. Swaan, Camptothecins: a review of their chemotherapeutic potential. Drugs, 2002. 62(14): p. 2039-57.

45. Sirianni, R.W., et al., Radiolabeling of Poly(lactic-co-glycolic acid) (PLGA) Nanoparticles with Biotinylated F18 Prosthetic Groups and Imaging of Their Delivery to the Brain with Positron Emission Tomography. Bioconjugate chemistry, 2014. 25(12): p. 2157-65. 
46. Zhou, J., et al., Highly penetrative, drug-loaded nanocarriers improve treatment of glioblastoma. Proceedings of the National Academy of Sciences of the United States of America, 2013. 110(29): p. 11751-6.

47. Xu, P., et al., Intracellular drug delivery by poly(lactic-co-glycolic acid) nanoparticles, revisited. Molecular pharmaceutics, 2009. 6(1): p. 190-201.

48. Bastiat, G., et al., A new tool to ensure the fluorescent dye labeling stability of nanocarriers: a real challenge for fluorescence imaging. Journal of controlled release : official journal of the Controlled Release Society, 2013. 170(3): p. 334-42.

49. Chen, H., et al., Release of hydrophobic molecules from polymer micelles into cell membranes revealed by Forster resonance energy transfer imaging. Proceedings of the National Academy of Sciences of the United States of America, 2008. 105(18): p. 6596-601.

50. Gullotti, E. and Y. Yeo, Beyond the imaging: limitations of cellular uptake study in the evaluation of nanoparticles. Journal of controlled release : official journal of the Controlled Release Society, 2012. 164(2): p. 170-6. 


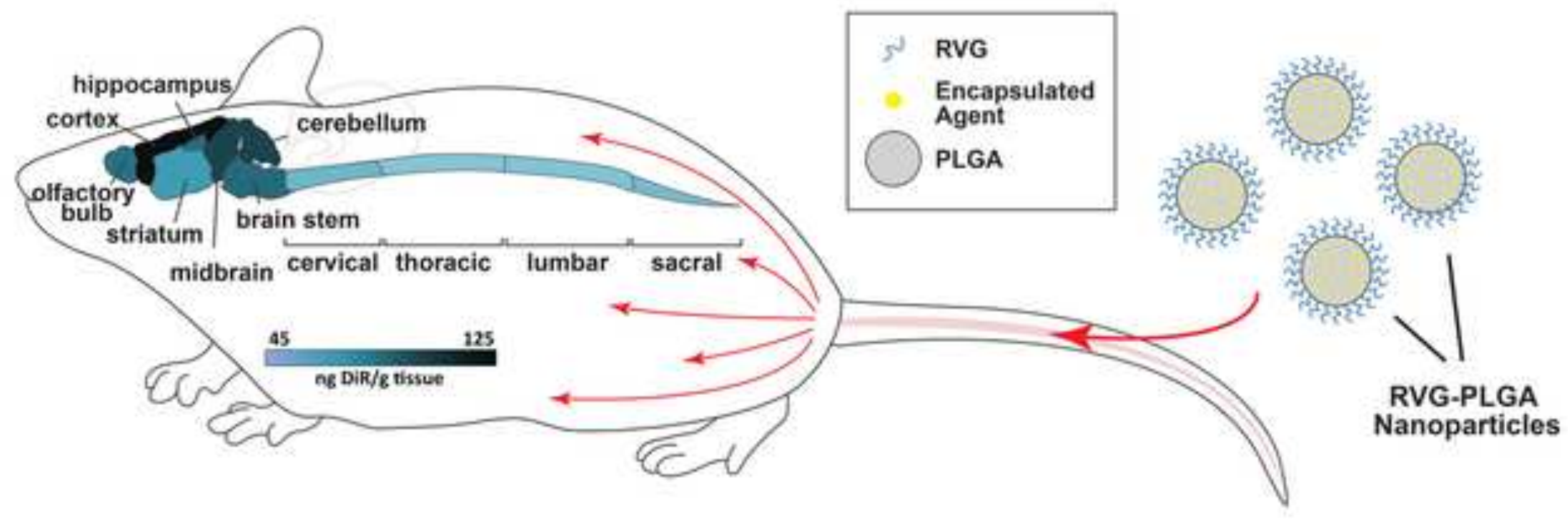

